\title{
Eficiencia del biochar obtenido a partir de residuos orgánicos municipales para la remoción de materia orgánica en aguas residuales, Chachapoyas, Amazonas, 2018.
}

\section{Efficiency of the biochar obtained from municipal organic waste for the removal of organic matter in wastewater, Chachapoyas, Amazonas 2018.}

\author{
Irina Ramirez Mas ${ }^{1 *}$, Oscar Andrés Gamarra Torres ${ }^{1}$, Manuel Emilio Milla Pino ${ }^{1}$ \\ RESUMEN
}

La materia orgánica municipal que se genera en las viviendas del Perú gran parte de estos residuos orgánicos no tienen un tratamiento adecuado, dentro de los residuos sólidos el $60 \%$ del total es materia orgánica, por lo que representa un bio recurso potencial para darle otros usos como el biochar. En este contexto se investigó la eficiencia del biochar, obtenido a partir de residuos orgánicos, para remover la materia orgánica del agua residual, para esto se produjo biochar mediante pirólisis a una temperatura 550 a $660^{\circ} \mathrm{C}$ en un horno pirolítico de doble tambor. Para el tratamiento del agua residual de la quebrada Santa Lucía de la ciudad de Chachapoyas, se empleó un filtro cuyo componente principal fue el biochar, arena fina, arena gruesa y piedras de río. La eficiencia máxima del filtro con biochar en la remoción de materia orgánica de la aguas residuales de la quebrada de Santa Lucía fue de $75 \%$ en remoción de DBO (en el experimento $\mathrm{N}^{\circ} 2$ ) y de $71 \%$ de remoción de DQO (en el experimento $\mathrm{N}^{\circ} 6$ ), debido a que en dichos experimentos se utilizó un biochar con una composición de tuza de maíz de 90 y $45 \%$ respetivamente. Los hallazgos de este estudio pueden ser mejorados ya que la eficiencia del biochar depende de la porosidad y de las características de la biomasa empleada para su síntesis.

Palabras claves: biochar, filtración de aguas residuales, pirolisis.

\begin{abstract}
The municipal organic matter that is generated in homes in Peru, a large part of this organic waste does not have an adequate treatment, within the solid waste $60 \%$ of the total is organic matter, so it represents a potential bio resource to give other uses like the biochar. In this context, the efficiency of biochar, obtained from organic waste, was investigated to remove the organic matter from the residual water, for which biochar was produced by pyrolysis at a temperature of 550 to $660^{\circ} \mathrm{C}$ in a double-drum pyrolytic furnace. For the treatment of wastewater from the Santa Lucía creek in the city of Chachapoyas, a filter was used whose main component was biochar, fine sand, coarse sand and river stones. The maximum efficiency of the biochar filter in the removal of organic matter from the wastewater of the Santa Lucía creek was $75 \%$ in BOD removal (in experiment $\mathrm{N}^{\circ}$. 2) and $71 \%$ removal of $\mathrm{COD}$ (in the experiment $\mathrm{N}^{\circ}$. 6), due to the fact that in these experiments a biochar with a corn gum composition of 90 and $45 \%$ was used respectively. The findings of this study can be improved since the efficiency of the biochar depends on the porosity and the characteristics of the biomass used for its synthesis.
\end{abstract}

Keywords: biochar, sewage filtration, pyrolysis.

${ }^{1}$ Facultad de Ingeniería Civil y Ambiental de la Universidad Nacional Toribio Rodríguez de Mendoza de Amazonas Amazonas - Perú

*Autor para correspondencia, e-mail: irina.ramirez@untrm.edu.pe 


\section{INTRODUCCIÓN}

En la actualidad, se genera una gran cantidad de residuos sólidos por crecimiento poblacional, económico y un estilo de vida orientado al consumo excesivo (Taherymoosavi, Verheyen, Munroe, Joseph, \& Reynolds, 2017). A medida que crece la urbanización, la cantidad de residuos sólidos municipales también se incrementa, incluso más rápido que la tasa de urbanización. Hace diez años se generaban alrededor de 0,68 mil millones de toneladas de residuos sólidos por año, hoy en día se genera 1,3 mil millones de toneladas por año. Para el año 2025 , se estima que se generará 2,2 billones de toneladas por año (Hoornweg \& Bhada-Tata, 2012).

La gestión ambientalmente racional de los desechos municipales se ha convertido en un desafío mundial debido a la limitada disponibilidad de recursos, al aumento de la población, especialmente en los países en desarrollo, a una urbanización e industrialización sin precedentes e irreversibles. Varios países se han dado cuenta de la forma en que manejan sus desechos sólidos no satisface los objetivos del desarrollo sostenible (Ramachandra, Bharath, Kulkarni, \& Han, 2018).

En el Perú, la generación de residuos sólidos municipales es aproximadamente de 7 millones toneladas por año, de los cuales 4 millones de toneladas son dispuestos en botaderos $\mathrm{u}$ otros destinos no identificados (Ministerio del Ambiente, 2017). Esta generación de residuos sólidos está provocando numerosos problemas ambientales (Taherymoosavi et al., 2017), como por ejemplo las emisiones de gases de efecto invernadero (GEI), contaminación del agua y el suelo afectando a la flora, fauna y a la salud pública (Mia et al., 2018). Además, el incorrecto manejo de los residuos orgánicos provoca malos olores, vectores, roedores y lixiviados que pueden contaminar a las fuentes de agua superficial y subterránea (Reddy \& Nandini, 2011).

Para superar los problemas mencionados anteriormente, la valorización de los residuos es una opción técnica que se está impulsado en la actualidad (Zabaleta \& Rodic, 2015, Xiao et al., 2017). En este contexto la producción de biochar, el cual es obtenido por conversión termoquímica de biomasa, como residuos agrícolas, astillas de madera, estiércol y residuos sólidos municipales (Agegnehu, Srivastava, \& Bird, 2017).

Las aplicaciones del biochar son muy diversas, desde la producción de calor y energía, limpieza de gases de combustión, aplicaciones metalúrgicas, uso en agricultura y cría de animales, material de construcción, para uso médico. Por ello, el biochar ha atraído un gran interés científico para aplicaciones agrícolas y medioambientales ya que tiene propiedades ventajosas para la reducción de la contaminación del suelo y agua (Vikrant et al., 2018). El biochar se caracteriza por una gran superficie específica, porosidad, cantidad de grupos funcionales y fácil obtención (Godlewska, Schmidt, Ok, \& Oleszczuk, 2017), por lo cual, el biochar se puede usar como un adsorbente de bajo costo para el tratamiento de aguas residuales (Oliveira et al., 2017).

Por lo tanto, el objetivo de la presente investigación fue evaluar la eficiencia del biochar obtenido a partir de residuos orgánicos municipales para la remoción de materia orgánica en aguas residuales, Chachapoyas, Amazonas, 2018. 


\section{MATERIAL Y MÉTODOS}

\subsection{Zona de estudio}

Se caracterizó los residuos orgánicos municipales que produce una vivienda en la ciudad de Chachapoyas con coordenadas $6^{\circ} 13^{\prime} 57.38^{\prime \prime} \mathrm{S}$ y 77²51'34.30' para conocer la cantidad de desechos orgánicos que generan, los que se pueden emplear para producir biochar. La ubicación del punto de muestro del agua residual fue en las coordenadas $6^{\circ} 13^{\prime} 25.08^{\prime \prime} \mathrm{S}$ y $77^{\circ} 51^{\prime} 40.59^{\prime \prime O}$ de la quebrada de Santa Lucía en la ciudad de Chachapoyas.

\subsection{Metodología}

\subsubsection{Selección de la materia orgánica}

Con las inspecciones hechas a los residuos sólidos de la vivienda y la revisión bibliográfica realizada al Estudio de caracterización de residuos sólidos de Chachapoyas 2012; el tipo de residuo que más se genera es del tipo orgánico en un $68,60 \%$, el que está compuesto principalmente por: Cáscara de papa, yuca, plátano, piña, mandarina; restos de arroz, tomate, cáscara de huevos, de limón, y de cebolla, cáscaras de frijol, arverja, tallo de repollo, tuza de maíz, trozos de yuca deteriorada.

\subsubsection{Producción del biochar}

En la vivienda, la cantidad de materia orgánica recolectada dependió de la generación de esta. Se recogió $4 \mathrm{~kg}$ de materia orgánica fresca, luego se colocó en un lugar bajo techo a temperatura ambiente por 5 días para reducir la humedad antes de ponerla en el horno pirolítico.

Se diseñó el horno pirolítico para producir biochar en pequeñas cantidades, compuesto por dos tambores cilíndricos y la capacidad del horno fue de 4 a $5 \mathrm{~kg}$ de biomasa. El tambor cilíndrico pequeño: es la cámara interna del horno y sus dimensiones son de $20 \mathrm{~cm}$ de diámetro y $30 \mathrm{~cm}$ de alto, con volumen de $9425 \mathrm{~cm}^{3}$, y el tambor cilíndrico grande: es la cámara para combustión externa que contiene al tambor cilíndrico pequeño. Sus dimensiones son 50 $\mathrm{cm}$ de diámetro y $50 \mathrm{~cm}$ de alto con un volumen de $0,4 \mathrm{~m}^{3}$.

Las muestras de residuos orgánicos recolectados y parcialmente secos, fueron mezcladas $y$ posteriormente se pesó cada tipo de materia orgánica antes de colocarlos en el cilindro pequeño.

Por cada producción de biochar se controlaron las entradas del aire para ambos tambores, ya que el proceso de pirólisis se lleva a cabo con oxígeno limitado.Se colocó aproximadamente $4 \mathrm{~kg}$ de materia orgánica como son cáscaras de papa, frijol, tuza, yuca, tomates, limón, etc. luego se llenó completamente sin compactar el tambor interno y se aseguró con la tapa. El espacio entre los dos tambores fue llenado con materiales combustibles como son leña, ramas secas, bagazo, entre otros. Luego, se roció con ron de quemar y de prendió fuego al material combustible cuidadosamente, para iniciar la pirolisis, Este proceso duró aproximadamente 2 a 2,5 horas y el proceso de enfriamiento aproximadamente 3 horas, luego se retiró la cámara interna con biochar resultante. El biochar obtenido se lavó con agua destilada, empleando aproximadamente 15 litros para un kilogramo de biochar; con la finalidad eliminar las sustancias de color oscuro adheridas al biochar.

\subsubsection{Ensamblaje y construcción del filtro}

Se ensambló un filtro casero para tratar el agua residual de la quebrada Santa Lucía, con la finalidad de reducir la materia orgánica. Para el ensamble del filtro se utilizó una botella de Policarbonato de $20 \mathrm{~L}$, con las siguientes dimensiones internas: diámetro de $26 \mathrm{~cm}$ y altura de $42 \mathrm{~cm}$, en su interior se conformó un lecho filtrante compuesto por 5 capas: una en el 
fondo de $5 \mathrm{~cm}$ de algodón, luego una de $10 \mathrm{~cm} \mathrm{de}$ biochar que pesaba $1 \mathrm{~kg}$, encima $5 \mathrm{~cm}$ de arena fina, $5 \mathrm{~cm}$ de arena gruesa, $6 \mathrm{~cm}$ de piedra de río de 1 ”. Sobre la capa superior se colocó una tela coladera que retuvo la mayoría de partículas gruesas.

\subsubsection{Aplicación del biochar en el filtro}

En la operación de filtración se observó que la función de la tela coladera fue impedir el paso de partículas grandes, sin embargo las partículas de menor tamaño lograron pasar debido a que su entretejido no es lo suficientemente estrecho. Para el análisis de DBO y DQO se extrajo muestras de 250 $\mathrm{mL}$ de agua residual de la quebrada Santa Lucía, que fue envasada en recipientes de plástico PET. Se recogió una muestra de agua residual por día, del mismo punto y fueron entregadas al laboratorio LABISAG. Luego se recolectó 20 litros de agua residual para filtrarla en el filtro con biochar estructurado como se muestra en la Figura 8. El caudal de alimentación del filtro fue de $5 \mathrm{~m}^{3} / \mathrm{s}$, por lo que los $20 \mathrm{~L}$ se filtraron en 4 minutos. Del filtrado se obtuvo una muestra para el análisis de DBO y DQO.

\subsubsection{Calculo de la eficiencia}

La eficiencia de la eliminación de contaminantes se calculó con la siguiente ecuación:

$$
E=\frac{V_{I N I C I A L}-V_{F I N A L}}{V_{I N I C I A L}} * 100
$$

Donde:

$\mathrm{V}_{\text {INICIAL }}=$ valor inicial del agua residual $\sin$ la aplicación del filtro con biochar $\mathrm{V}_{\mathrm{FINAL}}=$ valor final del agua residual con la aplicación del filtro con biochar

\section{III.RESULTADOS}

\subsection{Selección de la materia orgánica para la producción de biochar}

Las muestras de biochar fueron producidas a partir de $4 \mathrm{~kg}$ de residuos sólidos orgánicos, a una temperatura de 550 a $660{ }^{\circ} \mathrm{C}$ mediante pirolisis. El porcentaje de residuos orgánicos que se utilizó en la producción de biochar, se describe a continuación :.

Biochar 1: cascara de camote $(20 \%)$, cáscara de plátano $(20 \%)$, yuca (15\%), cáscara de mandarina (10\%), papa $(10 \%)$, zanahoria $(10 \%)$, tallo de repollo $(10 \%)$ y otros $(5 \%)$.

Biochar 2: tuza de maíz (90 \%) y cáscara de frijol $(10 \%)$

Biochar 3: cáscara de frijol (20\%), cáscara de arverja (20\%), cáscara de camote $(20 \%)$, tuza de maíz $(10 \%)$, cáscara de palta $(10 \%)$, cáscara de piña $(10 \%)$ y otros $(10 \%)$.

Biochar 4: cáscara de frijol (40\%), cáscara de yuca $(15 \%)$, cáscara de camote $(15 \%)$, cáscara de plátano $(10 \%)$ y otros $(20 \%)$.

Biochar 5: tuza de maíz (20 \%), cáscara de zanahoria (15\%), cáscara de cebolla (15\%), cáscara de frijol (15\%), cáscara de arverja $(15 \%)$ y otros $(20 \%)$

Biochar 6: tuza de maíz (45 \%), tallo de repollo (15 $\%)$, cáscara de plátano (15\%), cáscara de zanahoria $(10 \%)$, cáscara de piña $(10 \%)$ y cáscara de mandarina $(5 \%)$

Biochar 7: cáscara de arverja (40\%), cáscara de frijol $(15 \%)$, cáscara de yuca $(10 \%)$, cáscara de papa $(10 \%)$ y otros $(25 \%)$

El biochar resultante conserva la forma y tamaño de su materia prima original como se observa en las Figuras 1 y 2 ; sin embargo, otras materias orgánicas como la cáscara de frijol, arverja y de residuos de menor consistencia estructural, no conservaron la 
forma, en consecuencia, se obtuvo un biochar pulverizado.

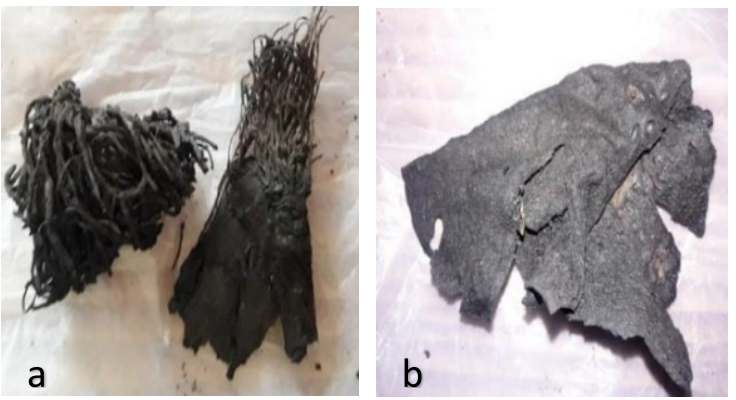

Figura 1. Muestras de biochar. a) Cáscara de cebolla. b) Cáscara de plátano.

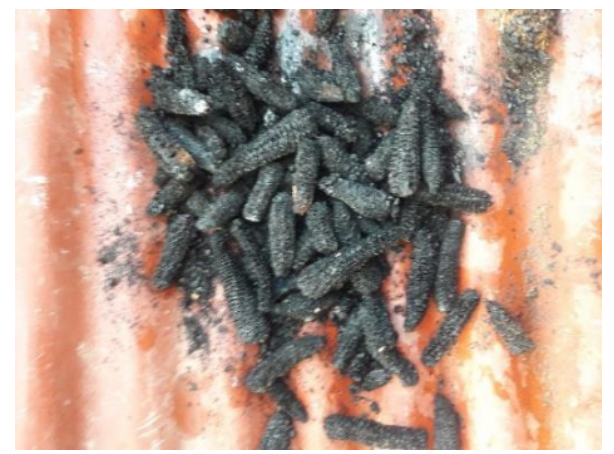

Figura 2. Muestra representativa de biochar de la tuza.

Al biochar de la cáscara de yuca y tuza de maíz se realizó una observación de poros a través de estereoscopio (SteREO very.V12) con una magnitud de 160.0 X y field (campo de observación) de 1.6 $\mathrm{mm}$; donde el biochar de la tuza de maíz (Figura 3) es más poroso que el biochar de cascara de yuca (Figura 4).

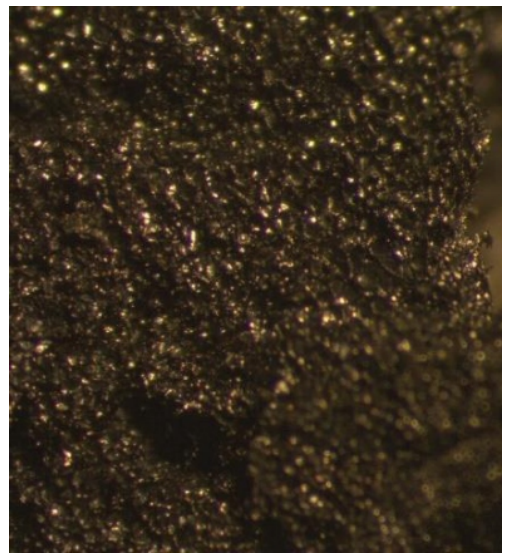

Figura 3. Poros del biochar de tuza de maíz

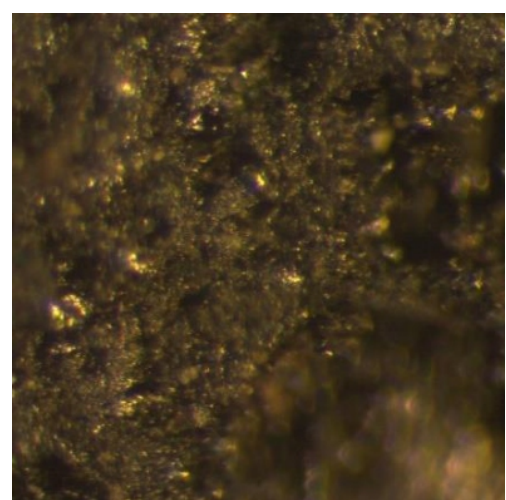

Figura 4. Poros del biochar de cáscara de yuca

\subsection{Eficiencia del biochar en el tratamiento de aguas residuales}

En la Figura 5, se muestra la eficiencia de remoción de DBO y DQO del filtro con biochar donde en las repeticiones 2 y 6 han tenido valores positivos de DBO (75 \% y $14 \%)$ y DQO (46 \% y $71 \%$ ) respectivamente.

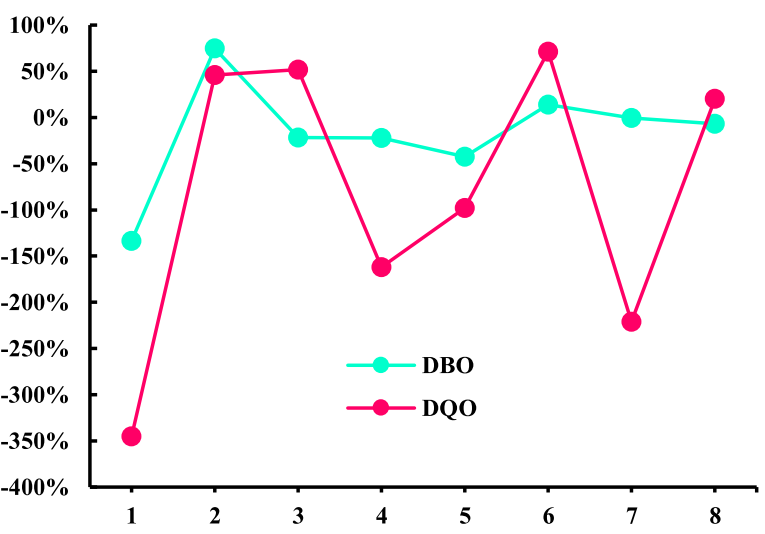

Figura 5. Eficiencia de remoción de DBO y DQO del filtro con biochar

\section{DISCUSIó N}

Los datos obtenidos en esta investigación permiten realizar un análisis de la eficiencia de la aplicación del filtro de biochar, que se realizó a nivel de laboratorio, el cual demostró que las características físicas de la materia orgánica es un factor determinante en la depuración de la misma, como se 
puede ver en la Figura 19 y 20 que la aplicación del filtro biochar para el agua residual no fue eficiente para remover la materia orgánica los 7 días, sin embargo como se puede observar en la Figura 19 y 20 hay 2 días en la que se realiza el efecto de remoción tanto de la DBO y DQO con un porcentaje remoción aceptable para el Límite Máximo Permisible para las PTAR, esto se debe que en esos días se colocó más biochar de tuza de maíz (presenta mayor porosidad y área superficial en consecuencia tiene mayor capacidad de retención) que de los otros biochar. Asimismo los resultados de la DBO evidencian una similitud antes y después de la aplicación del filtro de biochar en los 5 días, algunos valores más altos que otros, por lo que no se encuentra una diferencia significativa a la aplicación, pues aún falta acoplar o modificar al biochar para tener una remoción eficiente de la materia orgánica.

De los resultados de esta investigación, se aprecia que las propiedades físicas del biochar se vieron más afectadas por los tipos de residuos orgánicos empleados, por ejemplo; según estudios anteriores se estableció que los residuos de alimentos vegetales tienen una área de superficie más baja $\left(2-70 \mathrm{~m}^{2} / \mathrm{g}\right)$ mientras que los materiales leñosos tienen un área de superficie relativamente mayor $\left(200-380 \mathrm{~m}^{2} / \mathrm{g}\right)$, sin embargo el biochar derivado de la biomasa orgánica tuvo un rendimiento y un contenido de carbono relativamente bajo en comparación con el de la biomasa de madera, lo contrario sucede con las propiedades químicas del biochar orgánico que son relativamente mejores que las de los biochar de madera. Así la capacidad de retención de sustancias orgánicas depende de la materia prima según un estudio realizado por Liao, Liu, Li, \& Li, (2018).

Otro aspecto diferente que se encontró es que para tener una capacidad de adsorción del biochar de tuza de maíz, se debe realizar a diferentes temperaturas de pirolisis como por ejemplo $\left(350^{\circ} \mathrm{C}, 450^{\circ} \mathrm{C}\right.$ y 550 $\left.{ }^{\circ} \mathrm{C}\right)$ para poder ser modificado con otras compuestos químicos para mejorar la adsorción (Luo et al., 2018). Por otro lado, el biochar puede tener alta área de superficie, arquitectura flexible y alta porosidad para la reducción de la contaminación; sin embargo, el biochar tiene ciertos inconvenientes por ejemplo, capacidad limitada de sorción para los aniones y propiedades mecánicas deficientes que limitan su aplicabilidad práctica (Vikrant et al., 2018).

Sin embargo, para el futuro, el biochar sigue siendo un carbón prometedor para la remoción de materia orgánica, ya que muchas investigaciones tienen éxito para el tratamiento de aguas residuales. La experimentación es crucial para mejorar la sostenibilidad de la producción de alimentos, la incorporación del biochar es esencial para optimizar la sostenibilidad ambiental y económica de los residuos orgánicos (Dalahmeh, Ahrens, Gros, Wiberg, \& Pell, 2018).

\section{CONCLUSIONES}

La composición de los residuos orgánicos generados en la vivienda influyó en la eficiencia de remoción de materia orgánicas del agua residual de la quebrada de Santa Lucía, ya que, el tipo de consistencia y estructura delos residuos son parámetros que influyen en la porosidad del biochar.

La eficiencia máxima del filtro con biochar en la remoción de materia orgánica de la aguas residuales de la quebrada de Santa Lucía fue de $75 \%$ en remoción de DBO (en el experimento $\mathrm{N}^{\circ}$ 2) y de 71 $\%$ de remoción de DQO (en el experimento $\mathrm{N}^{\circ} 6$ ), debido a que en dichos experimentos se utilizó un biochar con una composición de tuza de maíz de 90 y $45 \%$ respetivamente. 
La producción de biochar es una alternativa sostenible de revaloración de residuos orgánicos domiciliares, además, puede ser utilizado como medio de tratamiento para la remoción de materia orgánica de las aguas residuales urbanas, combinado con otras técnicas de tratamiento de aguas residuales.

\section{REFERENCIAS BIBLIOGRÁFICAS}

Agegnehu, G., Srivastava, A. K., \& Bird, M. I. (2017). The role of biochar and biocharcompost in improving soil quality and crop performance: A review. Applied Soil Ecology, 119(April),156-170.

doi.10.1016/j.apsoil.2017.06.008

Dalahmeh, S., Ahrens, L., Gros, M., Wiberg, K., \& Pell, M. (2018). Potential of biochar filters for onsite sewage treatment: Adsorption and biological degradation of pharmaceuticals in laboratory filters with active, inactive and no biofilm. Science of the Total Environment, $612,192-201$

doi.10.1016/j.scitotenv.2017.08.178

Godlewska, P., Schmidt, H. P., Ok, Y. S., \& Oleszczuk, P. (2017). Biochar for composting improvement and contaminants reduction. A review. Bioresource Technology, 246(May), 193-202. doi.10.1016/j.biortech.2017.07.095

Hoornweg, D., \& Bhada-Tata, P. (2012). What a Waste: A Global Review of Solid Waste Management. Banco Mundial.

Liao, A. F., Liu, Y., Li, Q., \& Li, Y. (2018). SC. Journal of Analytical and Applied Pyrolysis. doi.10.1016/j.jaap.2018.08.001

Luo, M., Lin, H., Li, B., Dong, Y., He, Y., \& Wang, L. (2018). A novel modification of lignin on corncob-based biochar to enhance removal of cadmium from water. Bioresource Technology. doi.10.1016/j.biortech.2018.03.075
Mia, S., Uddin, M. E., Kader, M. A., Ahsan, A., Mannan, M. A., Hossain, M. M., \& Solaiman, Z. M. (2018). Pyrolysis and co-composting of municipal organic waste in Bangladesh: A quantitative estimate of recyclable nutrients, greenhouse gas emissions, and economic benefits. Waste Management. doi.10.1016/J.WASMAN.2018.01.038

Ministerio del Ambiente. (2017). PERÚ LIMPIO PERÚ NATURAL APROBACIóN DE LOS INSTRUCTIVOS 2017.

Oliveira, F. R., Patel, A. K., Jaisi, D. P., Adhikari, S., Lu, H., \& Khanal, S. K. (2017). Environmental application of biochar: Current status and perspectives. Bioresource Technology, 246, $110-122$ doi.10.1016/J.BIORTECH.2017.08.122

Ramachandra, T. V., Bharath, H. A., Kulkarni, G., \& Han, S. S. (2018). Municipal solid waste: Generation, composition and GHG emissions in Bangalore, India. Renewable and Sustainable Energy Reviews, 82, 1122-1136. doi.10.1016/J.RSER.2017.09.085

Reddy, P., \& Nandini, N. (2011). Leachate characterization and assessment of groundwater pollution near municipal solid waste landfill site. Journal of Environmental Monitoring and Assessment, 10, 415-418. doi.10.1007/s10661-006-1505-7

Taherymoosavi, S., Verheyen, V., Munroe, P., Joseph, S., \& Reynolds, A. (2017). Characterization of organic compounds in biochars derived from municipal solid waste.

Waste Management, 67, 131-142. doi.10.1016/J.WASMAN.2017.05.052

Vikrant, K., Kim, K.-H., Ok, Y. S., Tsang, D. C. W., Tsang, Y. F., Giri, B. S., \& Singh, R. S. (2018). Engineered/designer biochar for the removal of phosphate in water and wastewater. Science 
of The Total Environment, 616-617, 12421260.

doi.10.1016/J.SCITOTENV.2017.10.193

Xiao, R., Awasthi, M. K., Li, R., Park, J., Pensky, S. M., Wang, Q., ... Zhang, Z. (2017). Recent developments in biochar utilization as an additive in organic solid waste composting: A review. Bioresource Technology, 246, 203213. doi.10.1016/J.BIORTECH.2017.07.090

Zabaleta, I., \& Rodic, L. (2015). Recovery of essential nutrients from municipal solid waste - Impact of waste management infrastructure and governance aspects. Waste Management, $44,178-187$.

doi.10.1016/J.WASMAN.2015.07.033 